\title{
A inserção institucional do controle da doença de Chagas
}

\author{
Institutional Insertion of Chagas' disease control
}

\author{
Antônio Carlos Silveira* e Fabiano Pimenta Junior**
}

RESUMO

Em 1943, a partir da criação do "Centro de Estudos e Profilaxia da Moléstia de Chagas" da Fundação Oswaldo Cruz de Bambuí em Minas Gerais, são concebidas as bases tecnológicas e metodológicas para o controle extensivo da enfermidade. Para isso foi decisivo o advento de um novo inseticida (o gammexane, P 530) e a demonstração de sua eficácia no controle dos vetores da doença de Chagas. Como resultado prático desses acontecimentos em "maio de 1950 foi oficialmente inaugurada, em Uberaba, a primeira campanha de profilaxia da doença de Chagas, no Brasil". Mesmo que se dispusesse desde então de meios para fazer o controle da transmissão vetorial da endemia chagásica, não se dispunha dos recursos financeiros exigidos para fazê-lo de forma abrangente e regular. 0 baixo nível de prioridade conferida a essa atividade se expressava em sua inserção institucional. Em 1941, foram criados os Serviços Nacionais, de malária, peste, varíola, entre outros, enquanto a doença de Chagas fazia parte da Divisão de Organização Sanitária (DOS), que reunia enfermidades consideradas de menor importância. Em 1956 o Departamento Nacional de Endemias Rurais (DNERu) incorporou todas as chamadas grandes endemias em uma única instituição, mas na prática isso não significou a implementação das ações de controle da doença de Chagas. Com a reestruturação do Ministério da Saúde em 1970, a Superintendência de Campanhas de Saúde Pública (SUCAM) abarcou todas as endemias rurais, e a doença de Chagas passou a ter o status de Divisão Nacional, na mesma posição hierárquica daquelas outras doenças transmitidas por vetores antes consideradas prioritárias. Essa condição determinou a possibilidade de uma repartição de recursos mais equilibrada, o que efetivamente ocorreu, com a realocação de pessoal e insumos do programa de malária para o controle vetorial da doença de Chagas. Em 1991, a Fundação Nacional de Saúde sucedeu a SUCAM no controle das doenças endêmicas, congregando ademais todas as unidades e serviços do Ministério da Saúde relacionados à epidemiologia e ao controle de doenças. Já então a tendência era a descentralização operativa destes programas, o que no caso das doenças transmitidas por vetores representava uma drástica mudança no modelo campanhista até então vigente. À época, coincidentemente, foi formada a Iniciativa dos Países do Cone Sul para o controle da doença de Chagas, com o trabalho tecnicamente compartido entre os países da região, com metas e objetivos comuns, o que de algum modo contribuiu para que fosse preservada a doença de Chagas como prioridade entre os problemas de saúde. Desde 2003 as atividades de controle da doença no nível central nacional estão sob responsabilidade da Secretaria de Vigilância em Saúde do Ministério da Saúde.

Palavras-chaves: Doença de Chagas. Controle. História. Institucionalização do controle.

\begin{abstract}
After the starting of the Center for studies and prophylaxis of Chagas disease in 1943, with the help of Oswaldo Cruz Foundation, in the city of Bambuí, state of Minas Gerais, technological and methodological basis for the extensive control of the disease were conceived. A main step to achieve success was the introduction of a new insecticide (gammexane, P 530) and the demonstration of its efficacy in the vector control. A consequence of these improvements was the official inauguration of the first prophylactic campaign for Chagas disease in Brazil, held in Uberaba in May, 1950. Even with the knowledge of how to control the vectorial transmission, financial resources were not available by this time, at a necessary degree to make it both regularly and in all the affected area. The institutional allocation of these activities is useful to understand the low priority given to them at that time. Several national services were created in 1941, for diseases as malaria, pest, smallpox, among others, but Chagas was included in a group of diseases with lower importance, inside a Division of Sanitary Organization. In 1956, the National Department of Rural endemies (DNERu) allocate all the major endemic diseases in a single institution, however this was not translated in an implementation program for the control of Chagas disease. After profound changes at the Ministry of Health, in 1970, the Superintendência de Campanhas de Saúde Pública (SUCAM) was in charge of all rural endemies including Chagas disease, which now could compete with other diseases transmitted by vectors, formerly priorities, included in the National Division. With this new status, more funds were available, as well as redistribution of personnel and expenses from the malaria program to the vectorial control of Chagas disease. In 1991 the Health National foundation was created to substitute SUCAM in the control of endemic diseases and it included all the units of the Ministry of Health related to epidemiology and disease control. By this time a new tendency for decentralization of these programs was clear. In the case of diseases transmitted by vectors, this was a major difference from the campaign model so far employed. At the same time, the Initiative for the South Cone countries for the control of Chagas disease started, sharing techniques among the countries of this region, as well as establishing similar objectives and trends, what possible helped to maintain Chagas disease as a priority among all the public health issues. From 2003 on, all activities for control of the disease at a national level are under responsibility of the Secretary of Health Surveillance of the Ministry of Health.
\end{abstract}

Key-words: Chagas disease. Control. History. Control institutionalization.

\section{A INSTITUCIONALIZAÇÃO DO CONTROLE: ANTECEDENTES E CONTEXTO HISTÓRICO}

A inserção institucional das ações de controle da doença de Chagas nas estruturas de governo refletiram, de algum

\footnotetext{
*Ex-Diretor da Divisão Nacional de Doença de Chagas e da Divisão Nacional de Epidemiologia do Ministério da Saúde e Consultor da Organização Panamericana de Saúde.

**Secretaria Municipal de Saúde de Belo Horizonte, Minas Gerais.

Endereço para correspondência: Dr. Antonio Carlos Silveira. SQN 304/B/302 70736-020 Brasília, DF, Brasil.

e-mail: atcrs@uol.com.br
}

modo, a cada tempo, a prioridade conferida a essas atividades. $\mathrm{O}$ que, por sua vez, foi determinado em alguma medida pelo contexto político e projetos de desenvolvimento econômico e social vigentes no país.

Até que fosse implementado, na forma de programa de alcance nacional e com recurso bastante para uma atuação continuada e com a abrangência necessária, o controle da endemia chagásica tardou em demasia, mesmo depois de se haver conhecido tecnologia e métodos comprovadamente eficazes de combate aos vetores domiciliados.

Com o advento da microbiologia como ciência, o final do século XIX e inicio do século XX correspondeu a um período 
de intensa produção de conhecimento sobre a etiologia e a epidemiologia das doenças transmissíveis. Muitos foram os agentes infecciosos, vetores, reservatórios e mecanismos de transmissão identificados ${ }^{1}$. No Brasil é de se destacar, entre outros acontecimentos, a descoberta da doença de Chagas.

O controle institucionalizado das chamadas enfermidades endêmicas, ou grandes endemias, tem como marco inicial o ano de 1916 quando são criados os primeiros Postos de Saneamento e Profilaxia Rural na periferia da Cidade do Rio de Janeiro ${ }^{2}$.Representam o modelo de atuação que orienta e que dá origem em 1918 ao Serviço de Profilaxia Rural. A partir de então, com cooperação da Fundação Rockefeller, são instalados postos em outros estados, nas capitais e cidades do interior.

Importa notar que menos de uma década após a descrição da doença de Chagas havia estruturas permanentes de saúde no país dedicadas ao controle de doenças. E note-se ainda, especialmente, que o próprio Carlos Chagas foi um dos mentores da criação dos Postos de Saneamento e Profilaxia Rural, e um dos responsáveis por sua expansão: Este 'projeto', que priorizava o combate a doenças como a malária, a ancilostomose e a doença de Chagas, expande-se e atinge diversos pontos do interior do país, durante a gestão de Carlos Chagas ${ }^{2,3}$.

Essas três enfermidades eram apontadas pelos "cientistas e intelectuais do 'movimento sanitarista dos anos 10' como grande obstáculo ao progresso nacional, a ser superado mediante o compromisso do Estado em promover o saneamento rural do país" 4,5 .

Nas décadas de 1900 e 1910, a estrutura do Estado experimentou forte expansão, com o aumento do poder controlador do governo federal, através de instrumentos como a Justiça Sanitária, com a aprovação da vacinação obrigatória contra a varíola e o estabelecimento de notificação compulsória para várias doenças. No entanto estas políticas tinham aplicação limitada ao Distrito Federal e aos portos do país ${ }^{4,6}$.

Em 1919, o novo presidente da república, Epitácio Pessoa, declara em sua posse que os serviços sanitários são uma prioridade nacional. Naquele mesmo ano, Carlos Chagas é nomeado Diretor Geral de Saúde Pública e, no ano seguinte, com a criação do Departamento Nacional de Saúde Pública (DNSP), subordinado ao Ministério da Justiça e Negócios Interiores, assume a direção do novo órgão.

O DNSP nasce na perspectiva de buscar a centralização administrativa, ampliando a regulação do governo federal na saúde pública. Chagas em palestra proferida na Biblioteca Nacional, em fevereiro de 1921, expressa os propósitos da reforma pretendida. No referente ao controle das doenças endêmicas afirma que: "... No actual programma dos serviços sanitarios do Brasil figura o combate ás endemias ruraes, como um dos seus principaes escopos. Dous aspectos essenciaes do problema foram ahi considerados e fixados em dispositivos de lei: o technicoadministrativo e o financeiro. No primeiro de taes aspectos houve o governo de orientar-se pelo criterio preferivel de maxima centralização possivel daquelles serviços, de modo que, sem diminuir a autononia administrativa dos Estados da União, fosse nelles exercitada, com toda a amplitude, a applicação das medidas de saneamento e prophylaxia; e para conseguir que assim seja, os Estados, antes de iniciados os serviços, nos seus territorios, obrigam-se a aceitar e fazer com que sejam aceitas pelos municipios, as leis federaes concernentes ao assumpto. Não custa justificar a necessidade absoluta daquella centralização, determinada nos regulamentos federaes. Em primeiro lugar é de admittir que a maior capacidade technica em taes assumptos pertença á União; e se por ventura alguns Estados a possuem igual, constituem elles raras excepções, as quaes não poderiam actuar no espirito da lei..."

Em seu discurso, não faz nenhuma referência explícita à doença de Chagas. Duas condições limitavam então as possibilidades de controle da enfermidade, e que se estenderam pelos anos 20 e 30. Por um lado, a importância e magnitude da doença são postas em dúvida e, até mesmo, sua existência como entidade nosológica. Por outra parte, sua limitada vulnerabilidade, pela inexistência de instrumentos de controle de natureza estritamente sanitária. Os meios disponíveis de controle da transmissão dependiam fundamentalmente da melhoria das condições de habitação da população rural sob risco.

Carlos Chagas já em 1918 anunciava que "A prophylaxia da nova doença consta essencialmente do combate ao insecto transmissor". No entanto, pouco se havia evoluído até o final da década de 30 no desenvolvimento de técnicas e métodos para seu controle, aplicáveis em larga escala. Nem mesmo a distribuição dos vetores era conhecida.

Citados por Pellegrino ${ }^{7}$ Martins \& Tupinambá em 1940 justificavam a "...falta de publicações sobre a esquizotripanose, fora de Lassance, não só no longo período durante o qual foram se desenvolvendo as investigações naquela zona, mas também muito tempo depois, pelo descredito em que cahiu entre nós a moléstia de Chagas"; e, citando Laranja ${ }^{8}$ : "O cepticismo generalizado quanto à realidade das formas clínicas crônicas, orientou a pesquisa da moléstia para os casos de infecção aguda,nos quais a facilidade da demonstração do parasito no organismo permitia deduções etiopatogênicas seguras para as manifestações clínicas encontradas". E Pellegrino concluía que "Para isso muito contribuiu a descrição

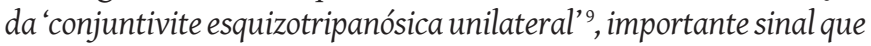
ocorre em grande número de casos de infecção recente, e denominado por Dias e E. Chagas, sinal de Romaña".

No que respeita ao ordenamento institucional, o Departamento Nacional de Saúde Pública desde novembro de 1930 passa a ser parte do recém criado Ministério da Educação e Saúde Pública (MESP). Sua criação foi um dos primeiros atos do Governo Provisório de Getúlio Vargas. O MESP integrou repartições que pertenciam aos Ministérios da Justiça e Negócios Interiores, da Viação e Obras Públicas, Agricultura e Indústria e Comércio. Compreendia de início quatro departamentos nacionais: Ensino, Saúde Pública, Medicina Experimental - ao qual estava ligado o Instituto Osvaldo Cruz - e Assistência Pública.

A partir dos anos 40, são criadas as condições iniciais para que a doença de Chagas passe a ser considerada problema de saúde pública, no país e no continente. São superadas as "dúvidas" relativas a enfermidade, sua magnitude e gravidade. Ademais, se comprova a eficácia de técnicas e métodos de controle com o uso sistematizado de inseticidas de ação residual em habitações infestadas pelo vetor.

\section{OS SERVIÇOS NACIONAIS E A DIVISÃO DE ORGANIZAÇÃO SANITÁRIA}

O decreto-lei n. 3.171 de 2 de abril de 1941 "Reorganiza o Departamento Nacional de Saúde do Ministério da Educação e Saúde e dá outras providencias". Em seu artigo $2^{\circ}$ estão 
discriminadas as unidades que passam a compor a estrutura do DNSP:

$\begin{array}{ll}\text { I. } & \text { Serviço de Administração } \\ \text { II. } & \text { Divisão de Organização Sanitária } \\ \text { III. } & \text { Divisão de Organização Hospitalar } \\ \text { IV. } & \text { Instituto Osvaldo Cruz } \\ \text { V. } & \text { Serviço Nacional de Lepra } \\ \text { VI. } & \text { Serviço Nacional de Tuberculose } \\ \text { VII. } & \text { Serviço Nacional de Febre Amarela } \\ \text { VIII. } & \text { Serviço Nacional de Malária } \\ \text { IX. } & \text { Serviço Nacional de Peste } \\ \text { X. } & \text { Serviço Nacional de Doenças Mentais } \\ \text { XI. } & \text { Serviço Nacional de Educação Sanitária } \\ \text { XII. } & \text { Serviço Nacional de Fiscalização da Medicina } \\ \text { XIV. } & \text { Serviço de Saúde dos Portos } \\ \text { XIV. } & \text { Serviço Federal de Águas e Esgotos } \\ \text { XV. } & \text { Serviço Federal de Bioestatística } \\ \text { XVI. } & \text { Sete Delegacias Federais de Saúde }\end{array}$

As prioridades, como se pode notar, expressas na nova estrutura do DNSP, correspondiam a enfermidades clinicamente evidentes, quase sempre agudas, de ocorrência predominantemente urbana e algumas delas com transmissão epidêmica. Entre os Serviços Nacionais, não constava a doença de Chagas. Distintamente daquelas, uma doença com pouca ou nenhuma aparência clínica na fase aguda, de evolução crônica, afetando grupos populacionais rurais, econômica e socialmente pouco influentes. Além disso, até então, era pouco vulnerável ao controle, pela inexistência de recursos técnicos para intervenções de largo alcance.

As atividades de vigilância e prevenção da doença de Chagas, e de outras com características e situação similares (esquistossomose), ou consideradas de menor importância (bouba, tracoma, leishmanioses), eram coordenadas e gerenciadas no nível central nacional pela Divisão de Organização Sanitária.

Com a criação pela Fundação Oswaldo Cruz, em novembro de 1943 , do "Centro de Estudos e Profilaxia da Moléstia de Chagas" (CEPMC) em Bambuí, Minas Gerais, dirigido por Emanuel Dias, passaram a ser ensaiadas diferentes alternativas para o controle da transmissão vetorial da doença de Chagas. $\mathrm{Na}$ revisão publicada por Pellegrino "A doença de Chagas em Minas Gerais; Esbôço crítico dos trabalhos publicados até 1951" ", consta que: "Logo em seguida à instalação do Centro foi organizado um plano de combate à esquizotripanose. Em relatório apresentado ao Diretor do Instituto Oswaldo Cruz, reunindo os resultados dos trabalhos realizados nos seis primeiros meses de atividade Dias assim encara as linhas gerais de um plano de profilaxia da moléstia de Chagas: 1)combate direto aos vectores intermediários;2) combate aos portadores vertebrados de Schizotrypanum cruzi; 3) melhoria das habitações rurais; 4) educação sanitária".

Entre as possibilidades consideradas para o combate direto aos vetores vários inseticidas de contato e repelentes foram testados: querozene; gás cianídrico; piretro (fly-tox); DDT. E, segundo Pellegrino, Nenhum dos métodos mostrou-se realmente eficaz ${ }^{7}$.

A essas tentativas se seguiu, a experimentação de um novo inseticida, o hexaclorociclohexano, ou BHC ("gammexane,
P 530"), que em ensaios de laboratório e trabalhos de campo, conduzidos por Dias e Pellegrino a partir de 1947, se mostrou altamente tóxico para triatomíneos ${ }^{10}$.

No ano de 1948, animado com esses resultados, Pellegrino teria enviado telegrama ao então Ministro da Educação e Saúde, Clemente Mariani Bittencourt, informando, textual e laconicamente: controle da doença de Chagas tecnicamente resolvido ${ }^{11}$.

Como consequência prática dessa iniciativa e do trabalho de investigação realizado "no dia 7 de maio de 1950 foi oficialmente inaugurada, em Uberaba, a primeira campanha de profilaxia da doença de Chagas, no Brasil, para a realização da qual o Serviço Nacional de Malária (SNM) mobilizou numeroso pessoal habilitado e grande cópia de material. A região escolhida para o desenvolvimento desta campanha compreendeu 123 municípios do Estado de Minas Gerais e 93 outros do Estado de São Paulo ${ }^{7}$. Esse acontecimento inaugura as ações institucionalizadas de controle da doença de Chagas no país, e a primeira instituição pública na área de serviços de saúde a assumir a tarefa é o Serviço Nacional de Malária, do Departamento Nacional de Saúde do Ministério da Educação e Saúde.

O SNM vinha já colaborando com o CEPMC em provas estendidas de campo em áreas dos municípios de Uberaba, Santa Juliana e Bambuí. A proposta, para que o SNM assumisse a execução de desinsetização domiciliar, em escala de campanha para o combate à doença de Chagas, foi expressa em moção encaminhada ao Ministro da Educação e Saúde pelos participantes do I Congresso Médico do Brasil Central e Triângulo Mineiro, realizado em Araxá em 1949. Daí, resultou acordo formal entre a direção da Fundação Oswaldo Cruz e do Serviço Nacional de Malária ${ }^{12}$.

\section{O DEPARTAMENTO NACIONAL DE ENDEMIAS RURAIS (DNERU)}

Com a Lei no 1.920 de 25 de julho de 1953, foi desdobrado o Ministério da Educação e Saúde em dois outros: Ministério da Saúde e Ministério da Educação e Cultura. O Ministério da Saúde passou a exercer as funções do Departamento Nacional de Saúde (DNS).

Passados três anos desde a criação do Ministério da Saúde, o Decreto 2.743 institui o Departamento Nacional de Endemias Rurais (DNERu) a quem, tal como enunciado incumbe "organizar e executar os serviços de investigação e promover o combate à malária, leishmaniose, doença de Chagas, peste, brucelose, febre amarela, esquistossomose, ancilostomose, filariose, hidatidose, bócio endêmico, bouba, tracoma e outras endemias existentes no país, cuja investigação e combate lhe forem especialmente atribuídas...". Por esse mesmo diploma legal é criado o Instituto Nacional de Endemias Rurais (INERu), tendo como atribuição precípua "realizar pesquisas e estudos sobre as endemias indicadas com a finalidade de ampliar o conhecimento das mesmas e aperfeiçoar os métodos profiláticos destinados a combatê-las".

Na mensagem presidencial anual de 1956 (Juscelino Kubitschek de Oliveira), é feita menção ao fato de que " $a$ centralização de atividades para o combate as endemias rurais deve ser considerada um grande avanço, pois cada doença não mais será 
tratada como um problema autônomo". O DNERu incorpora os Serviços Nacionais de Malária, Peste e Febre Amarela, além das enfermidades cujo controle estava a cargo da Divisão de Organização Sanitária ${ }^{13}$.

A relação biunívoca entre saúde e desenvolvimento, no sentido de que ela é a um só tempo produto e agente do desenvolvimento econômico é expressamente manifesto no mencionado documento. Estranhamente, no capítulo dedicado as "Doenças predominantes no Brasil", a doença de Chagas não foi incluída entre elas. São mencionadas, pela ordem, malária, bouba, tuberculose, bócio endêmico, verminoses, esquistossomose, doenças da nutrição, doenças gastrointestinais e sífilis ${ }^{13}$.

Ainda que a doença de Chagas tenha auferido um espaço institucional próprio ou formalmente reconhecido na estrutura do DNERu, na prática isso não se traduziu em um aporte regular e suficiente de recursos financeiros para sustentar intervenções duradouras e com ampla cobertura. As atividades de controle na era DNERu se mantiveram de forma irregular, dependendo da disponibilidade maior ou menor de recursos. Os resultados eram por isso mesmo precários, ou transitórios. Colocava-se em dúvida a eficácia do controle químico ou a estabilidade das respostas com ele logradas. Em oposição, equivocadamente, argumentava-se que resultados duradouros apenas seriam obtidos pela melhoria física das habitações.

Em que pese o propósito de integração, a Campanha de Erradicação da Malária (CEM) que substituiu o SNM, a princípio como parte do Departamento Nacional de Endemias Rurais (DENERu), a partir de 1965, passou a ser uma agência autônoma. Uma instituição exclusivamente dedicada ao cumprimento daquela meta, de erradicação, o que atendia ao recomendado pela Organização Mundial da Saúde(OMS) desde 1955, quando da sua XIV Assembléia Mundial ${ }^{14}$. Assim ocorreu também com a Campanha de Erradicação da Varíola (CEV) instituída no Brasil em 1966, como parte de Campanha Mundial liderada pela OMS , com o objetivo de interromper a transmissão da doença no país através da vacinação em massa da população; e que serviu, de forma decisiva, para implantação de um sistema de vigilância epidemiológica no país ${ }^{15}$.

Esse foi o tempo de grandes apostas na erradicação de enfermidades epidêmicas de grande transcendência e mundialmente dispersas. Isso restringia os recursos para o controle de outras enfermidades, tal como a doença de Chagas, própria dos confins latino-americanos, e que se cria pouco vulnerável ao controle.

\section{A SUPERINTENDÊNCIA DE CAMPANHAS DE SAÚDE PÚBLICA (SUCAM)}

Em maio de 1970 é criada a Superintendência de Campanhas de Saúde Pública (SUCAM), pela fusão do DNERu, Campanha de Erradicação da Malária (CEM) e Campanha de Erradicação da Varíola (CEV).

A doença de Chagas passa a ter o status institucional de Divisão, na mesma posição hierárquica de Malária, Febre Amarela e outras doenças transmitidas por vetores antes consideradas prioritárias. Essa condição determina, de início, que se estabeleça a possibilidade de negociação para uma repartição de recursos mais equilibrada.

São revistas as normas do programa e se dá início em 1975 a amplos inquéritos epidemiológicos, de soroprevalência e entomológico, abrangendo todo o país. Recursos excedentes do programa de malária nas regiões nordeste, sudeste e em parte do centro-oeste são re-alocados, em favor do programa de controle da doença de Chagas. A partir de 1983, se expandem as ações de controle vetorial, que alcançam progressivamente a toda área com risco de transmissão domiciliar.

\section{A FUNDAÇÃO NACIONAL DE SAÚDE (FUNASA)}

Em 1991, a Fundação Nacional de Saúde (FUNASA) passa a congregar todas as instituições vinculadas ao Ministério da Saúde relacionadas com a epidemiologia e o controle de doenças: Fundação Serviços de Saúde Pública (FSESP), parte das Secretarias Nacionais de Ações Básicas de Saúde (SNABS) e de Programas Especiais de Saúde (SNPES) e a SUCAM. Antes de qualquer intenção integradora, o que parece haver presidido, ao menos de inicio, a decisão de fazer esse novo rearranjo organizacional foi a lógica do estado mínimo ou do enxugamento do aparelho do estado. Cada uma das antigas instituições passou a ocupar um espaço próprio dentro daquela que havia sido criada ${ }^{14}$.

A SUCAM passou a constituir uma Coordenação de Controle de Doenças Transmitidas por Vetores (CCDTV), onde os programas individualizados por doença foram mantidos, na forma de gerências nacionais.

\section{O PROCESSO DE DESCENTRALIZAÇÃO E A SECRETARIA DE VIGILÂNCIA DA SAÚDE (SVS)}

O processo de descentralização das ações de prevenção e controle de doenças no Brasil foi concretamente efetivado a partir do ano de 2000, com a publicação da Portaria M.S 1399 de dezembro de 2002, que definiu e regulamentou as responsabilidades de cada uma das esferas de governo nessa área e estabeleceu o mecanismo de financiamento, na modalidade fundo a fundo.

A efetiva implantação do processo de descentralização induziu mudanças na organização interna da Fundação Nacional de Saúde, notadamente quanto a coordenação e execução das ações de controle das doenças transmitidas por vetores, até então sob a responsabilidade do Departamento de Operações (DEOPE) e que, a partir de 1999, passaram ao Centro Nacional de Epidemiologia, (CENEPI), que concentrou como atribuições a gestão do sistema nacional de vigilância em saúde. Tais atribuições compreendiam basicamente a coordenação nacional das ações de Vigilância em Saúde, com ênfase naquelas atividades que exigem simultaneidade nacional ou regional, como condição de êxito; a normatização técnica; a gestão dos sistemas de informação epidemiológica e o fornecimento de insumos estratégicos ${ }^{16}$. 
A focalização das responsabilidades da esfera federal, aliada à intensificação das epidemias de dengue, atribuídas pelos formadores de opinião como decorrentes do processo de descentralização, desencadeou uma série de discussões quanto a melhor forma de organização da esfera federal, culminando com a edição da Medida Provisória nํ33 , publicada no Diário Oficial da União de 07 de março de 2002, criando a Agência Federal de Prevenção e Controle de Doenças - APEC, que veio a ser rejeitada pelo Plenário da Câmara dos Deputados, em 17 de abril de $2002^{17}$.

O Sistema Nacional de Vigilância Epidemiológica (SNVE) foi estruturado em 1975, com a promulgação da Lei $\mathrm{N}^{\circ}$ 6.259, regulamentada pelo Decreto Presidencial $N^{\circ} 78.231^{18}$. Em função da limitada cobertura dos serviços de saúde no momento em que foi criado e de forma coerente com a situação epidemiológica prevalecente à época de sua criação, o SNVE atuava exclusivamente sobre as doenças transmissíveis, e estava exclusivamente a cargo dos governos federal e estaduais. Os municípios não exerciam qualquer papel na gestão do sistema de saúde ${ }^{19}$.

Com o decorrer dos anos, o Brasil passou por importantes mudanças em sua estrutura demográfica e em seu perfil epidemiológico, com a queda da fecundidade, o declínio persistente da mortalidade infantil e da mortalidade por doenças infecciosas, o incremento da expectativa de vida ao nascer e o aumento na intensidade e freqüência de exposição a modos de vida pouco saudáveis. Esses acontecimentos implicaram o aumento da ocorrência de doenças crônicas não-transmissíveis.

Por outro lado, a ocorrência de epidemias e pandemias por doenças emergentes ou re-emergentes fez com que a comunidade internacional demandasse o aprimoramento dos serviços de vigilância em saúde, especialmente da capacidade de resposta às emergências epidemiológicas, incluindo o aumento da sensibilidade para detecção de eventos relevantes. A globalização determinou o aumento da circulação de pessoas e mercadorias, estreitou as distâncias e o compartilhamento de agentes de doenças.

Nesse contexto, em 2003, o Ministério da Saúde reorganizou a área de epidemiologia e controle de doenças, com a extinção do Centro Nacional de Epidemiologia (CENEPI) e a criação da Secretaria de Vigilância em Saúde. Esta passou a reunir todas as atribuições do CENEPI e dos programas que integraram a extinta Secretaria de Políticas de Saúde: tuberculose, hanseníase, hepatites virais e as doenças sexualmente transmissíveis e AIDS $^{19}$.

Essa alteração correspondeu a uma importante mudança institucional, na perspectiva da afluência de todas as ações de vigilância, prevenção e controle de doenças em uma mesma estrutura, e da consolidação do processo de ampliação dos objetos de vigilância.

A adoção do conceito de vigilância em saúde caracteriza e expressa concretamente essa nova abordagem, mais ampla do que a tradicional prática de vigilância epidemiológica, tal como foi efetivamente construída no país, desde a década de 70, incluindo a vigilância: I) das doenças transmissíveis; II) das doenças e agravos não transmissíveis e seus fatores de risco; III) ambiental em saúde; e IV) da situação de saúde, que corresponde a uma das aplicações da área também correntemente designada análise de situação de saúde ${ }^{20}$.

$\mathrm{Na}$ atual Secretaria de Vigilância em Saúde, as ações de controle da doença de Chagas passaram a ser gerenciadas no âmbito da Coordenação de Doenças Transmitidas por Vetores e Antropozoonoses, integrante da Coordenação-Geral de Doenças Transmissíveis do Departamento de Vigilância Epidemiológica. Importa destacar que alguns dos programas de controle de doenças, como é o caso da malária e do dengue, têm a estrutura de uma Coordenação-Geral; e outras, como a tuberculose, hanseníase e hepatites virais, possuem estruturas de coordenações específicas. Essa distinção está diretamente relacionada às ações prioritárias do Plano Plurianual de Governo 2004-2007 e às metas prioritárias do Pacto pela Vida.

\section{O CAMINHO PERCORRIDO}

A partir da revisão histórica que se procurou fazer o que se comprova, e não poderia ser de outro modo, é que o programa nacional de controle da doença de Chagas, desconsiderando-se aqui as diferentes denominações que a ele se atribuiu ao longo do tempo, obedeceu às políticas de saúde vigentes em diferentes momentos dessa história. Além disso, o espaço institucional que ocupou foi, em termos hierárquicos, mais ou menos proeminente em função da importância conferida ao controle da doença.

De início, enquanto não reconhecida sua prioridade como problema de saúde publica, o controle da doença de Chagas fazia parte, ao lado de outras doenças endêmicas menores, porque crônicas e/ou focais, de uma Divisão Nacional. Quando as chamadas endemias rurais passaram a constituir um serviço nacional com espaço próprio (DNERu), ganhou o status de Programa Nacional diferenciado com o advento da Campanha de Controle da doença de Chagas; e assim se manteve, quando da criação da SUCAM, como Divisão Nacional, período em que as atividades de controle foram grandemente intensificadas.

Depois disso, com a progressiva descentralização operativa do programa, e o nível nacional tendo assumido como funções que lhe seriam próprias a normalização, seguimento, avaliação e a entrega de cooperação técnica a estados e municípios, o Programa de Controle da doença de Chagas passou a existir como Gerência Técnica em uma Coordenação que reúne segundo uma outra lógica, as antropozoonoses.

Em síntese, se pode afirmar que a inserção institucional do controle da doença de Chagas na esfera nacional passou da indiferenciação à particularização; e, daí à integração das ações de vigilância e controle a um grupo de doenças epidemiologicamente similares, não só pelo mecanismo de transmissão (vetorial), mas também pela sua origem ancestral.

\section{REFERÊNCIAS}

1. Silva LJ. Controle das endemias no Brasil e sua história. Cienc Cult 2003; 55: 44-47.

2. Chagas Filho C. Meu Pai. Rio de Janeiro: Editora FIOCRUZ; 1993.

3. Campos CEA. A organização dos serviços de Atenção Primária à Saúde no Brasil. Rev Bras Med Fam Com 2006; 2: 131-147. 
4. Hochman G. A era do saneamento: as bases da política de saúde pública no Brasil. São Paulo: HUCITEC/Anpocs; 1998.

5. Kropf SP. Ciência, saúde e desenvolvimento: A doença de Chagas no Brasil (1943-1962) Tempo 2005; 10: 107-124.

6. Marques EC. Saúde pública e construção do Estado na Primeira República. Rev Bras Ci Soc 1999; 14: 182-186.

7. Pellegrino JA. Doença de Chagas em Minas Gerais; Esbôço crítico dos trabalhos publicados até 1951. Mem Inst Oswaldo Cruz 1953; 51: 611-668.

8. Laranja FS. Evolução dos conhecimentos sôbre a cardiopatia da moléstia de Chagas: revisão crítica da literatura. Mem Inst Oswaldo Cruz 1949; 47: 604-670.

9. Romaña C. Acerca de un síntoma inicial de valor para el diagnóstico de forma aguda de la enfermedad de Chagas, la conjuntivitis esquizotripanósica unilateral. Misión de Estudios de Patología Regional Argentina. Publicación n² 22; 1935.

10. Dias E, Pellegrino J. Alguns ensaios com o "Gamexanne" no combate aos transmissores da doença de Chagas. Brazil Médico 1948; 62: 185-190.

11. Coura JR. $O$ falso dilema sobre a luta antivetorial e as perspectivas de controle da doença de Chagas no Brasil. BHC ou BNH? Cad Saúde Publ 1993; 9: 514-518.

12. Kropf SP. A primeira campanha de profilaxia da doença de Chagas no Brasil (Uberaba, 1950). Disponível em URL: http://www.fiocruz.br/chagas/cgi/ cgilua.exe/sys/start.htm?sid=61.

13. Brazilian Presidential Messages, 1890-1993. Disponível em URL: http:// brazil.crl.edu/bsd/bsd/u1338/contents.html.
14. Silveira AC, Rezende DF. Avaliação da estratégia global de controle integrado da malária no Brasil. Brasília: Organização Pan-Americana da Saúde; 2001.

15. Gazeta AAB, Carvalho DM, Tura LFR, Rosangela Gaze R. A campanha de erradicação da varíola no Brasil e a instituição do Sistema Nacional de Vigilância Epidemiológica. Cad Saúde Colet 2005; 13: 323 - 338.

16. Brasil 1999. Portaria n. 1.399, de 15 de dezembro de 1999. Regulamenta a NOB SUS/96 no que se refere às competências da União, Estados, Municípios e Distrito Federal, na área de Vigilância em Saúde, define a sistemática de financiamento e dá outras providências. Disponível em URL: http://portal.saude.gov.br/portal/saude/texto.

17. Brasil 2002. Medida Provisória no 33 de 2002. Dispõe sobre os Sistemas Nacionais de Epidemiologia, de Saúde Ambiental e de Saúde Indígena, cria a Agência Federal de Prevenção e Controle de Doenças - APEC, e dá outras providências. Disponível em URL:www.planalto.gov.br/ccivil_03/ Exm/2002/42-MPO-02.htm.

18. Brasil 1976. Decreto n. 78.231, de 12 de agosto de 1976. Regulamenta a Lei n. 6.259, de 30 de outubro de 1975. Disponível em URL: http://wwwt. senado.gov.br/legbras.

19. Conselho Nacional de Secretários de Saúde. Vigilância em Saúde. Coleção Progestores - Para entender a gestão do SUS. Brasília: CONASS; 2007.

20. Silva Júnior JB. Epidemiologia em serviço: uma avaliação de desempenho do Sistema Nacional de Vigilância em Saúde. Campinas, SP: 318 p. [Tese de doutorado]. Faculdade de Ciências Médicas, Universidade Estadual de Campinas; 2004 